\title{
Editorial
}

The Editorial Board of Earth, Planets and Space wishes to thank the following reviewers for their help in evaluating papers for the year 2005 .

\section{List of Reviewers for 2005}

\begin{tabular}{|c|c|c|c|}
\hline Abidin, H. Z. & Christensen, D. H. & Gudmundsson, A. & Imanishi, $\mathrm{K}$. \\
\hline Adams, D. & Chulliat, A. & Guio, $\mathrm{P}$. & Imoto, $\mathrm{M}$. \\
\hline Albert, J. & Constable, C. & Gulyaeva, T. & Ishibashi, K. \\
\hline Altamimi, Z. & Crosthwaite, P. & Haagmans, R. & Ishido, $\mathrm{T}$. \\
\hline Amm, $\mathrm{O}$. & Dermanis, A. & Haas, R. & Ishihara, T. \\
\hline Anazawa, K. & Detman, T. R. & Hagstrum, J. & Ishiyama, $\mathrm{T}$. \\
\hline Andersen, O. & Dong, D. & Hanada, H. & Ito, $\mathrm{T}$. \\
\hline Antolik, M. & Dongping, W. & Hannah Fok, M.-C. & Ito, Y. \\
\hline Aoi, S. & Dreger, D. & Hara, T. & Iwamori, H. \\
\hline Artru, J. & Dyment, J. & Harinarayana, $\mathrm{T}$. & Iwata, $\mathrm{T}$. \\
\hline Bakun, W. & Earle, G. D. & Hasemi, A. & Iyemori, $\mathrm{T}$. \\
\hline Balan, N. & Ebert, U. & Hashimoto, $\mathrm{C}$. & Jackson, A. \\
\hline Ballatore, $\mathrm{P}$. & Eccles, J. V. & Hashimoto, K. & $\mathrm{Ji}, \mathrm{C}$. \\
\hline Bargatze, L. & Eguchi, T. & Hashimoto, M. & Johnson, A. \\
\hline Barnes, J. & Ekstrom, G. & Hatanaka, Y. & Johnston, M. \\
\hline Benson, R. & Ellis, S. & Hatayama, K. & Jones, J. \\
\hline Berozo, G. & Evans, R. L. & Hattori, K. & Joseph, J. \\
\hline Bevan, R. J. & Everett, M. & Hayashi, Y. & Kadokura, A. \\
\hline Biagi, F. P. & Fagundes, P. R. & Heki, K. & Kagawa, T. \\
\hline Biggs, J. & Ferraccioli, F. & Hernández-Pajares, M. & Kagiyama, T. \\
\hline Bilitza, D. & Finn, C. & Hino, R. & Kaidzu, M. \\
\hline Bock, Y. & Fisher, M. A. & Hirahara, K. & Kakehi, Y. \\
\hline Boehnel, H. & Flueh, E. R. & Hirata, N. & Kamaya, N. \\
\hline Bondar, T. & Fok, M.-C. & Hisada, Y. & Kameyama, M. \\
\hline Borcherdt, R. D. & Foster, J. C. & Hobiger, T. & Kanamatsu, T. \\
\hline Bouin, M. P. & Francis, W. & Holme, R. & Kanasima, S. \\
\hline Brandon, A. & Freymueller, J. T. & Holota, P. & Kanda, W. \\
\hline Brocher, T. M. & Fujima, K. & Holzer, T. & Kasahara, J. \\
\hline Brodsky, E. E. & Fujimoto, $\mathrm{H}$. & Honda, R. & Katao, H. \\
\hline Brozena, J. M. & Fujita, M. & Horike, $\mathrm{M}$. & Kato, A. \\
\hline Buchert, S. C. & Fujiwara, H. & Hoshiba, M. & Kato, T. \\
\hline Burgmann, R. & Fukuda, Y. & Housen, B. & Katsumata, K. \\
\hline Burke, W. J. & Funiciello, R. & Houston, H. B. & Katsura, T. \\
\hline Cain, J. C. & Furuya, M. & Houvelin, J. & Kawasaki, I. \\
\hline Cande, S. & Fuselier, S. & $\mathrm{Hu}, \mathrm{J} .-\mathrm{C}$. & Kawase, H. \\
\hline Castillo-Negrete, D. & Futaana, Y. & Hysell, D. L. & Kido, M. \\
\hline Cattin, R. & Gagnon, K. & Ichiki, M. & Killern, R. \\
\hline Chadwel, D. & Ge, M. & Ichinose, G. A. & Kim, H. R. \\
\hline Chau, H. D. & Geist, E. L. & Ieda, A. & Kimata, F. \\
\hline Chave, A. D. & Gibson, G. & Igel, H. & Kind, R. \\
\hline Chen, H.-W. & Gilbert, D. & Iio, Y. & Kissling, E. H. \\
\hline Chi, P.-J. & Gokarn, S. G. & Ikeda, R. & Kita, N. \\
\hline Chia, Y. & Grandis, H. & Ikeda, Y. & Kobayashi, S. \\
\hline Chouteau, M. & Graves, R. W. & Imamura, $\mathrm{F}$. & Kodaira, S. \\
\hline
\end{tabular}


Koketsu, K.

Komazawa, M.

Konagai, K.

Konopliv, A.

Korepanov, V.

Korte, M.

Kosuga, M.

Kotze, P.

Koyaguchi, T.

Koyama, J.

Koyama, T.

Kreemer, C.

Kuge, K.

Kuramitsu, Y.

Kuramoto, S.

Kuwahara, Y.

Laakso, $\mathrm{H}$.

Langenheim, V. E.

Larson, K.

LeBlanc, T.

Lee, C.-C.

Lee, D. H.

Lee, S. M.

Lesur, V.

Li, X.

Li, Y.

Li, Y.-G.

Lin, C. H.

Linde, A. T.

Love, J. J.

Lowes, F.

Lowry, A. R.

Luehr, H.

Macmillan, S.

Madariaga, R.

Maezawa, K.

Mahajan, K. K.

Mai, M.

Makino, M.

Mandea, M.

Martinis, C.

Maruyama, $\mathrm{T}$.

Mathews, J. D.

Matsubayashi, H.

Matsumoto, K.

Matsumoto, S.

Matsumura, S.

Matsushima, M.

Matsushima, $\mathrm{T}$.

Matsutomi, $\mathrm{H}$.

Matsuyama, M.

Maus, S.

Maxwell, S.

McCrea, I.

McCreadie, H.

McDonald, F. B.

McLean, S. J.

Medvedev, M.

Miki, D.

Miller, K. C.
Mitchell, N. J.

Mitsuhata, Y.

Miura, S.

Miyake, H.

Miyake, W.

Miyauchi, T.

Miyazaki, S.

Mochizuki, N.

Mofjeld, H.

Mogi, T.

Moore, B.

Mori, J.

Morikawa, N.

Moriya, T.

Murai, Y.

Murakami, H.

Murayama, Y.

Murru, M.

Nagao, $\mathrm{H}$.

Nagao, $\mathrm{T}$.

Nagasawa, C.

Nakada, M.

Nakamichi, H.

Nakamura, K.

Nakanishi, M.

Nakano, S.

Nakatsuka, T.

Namiki, N.

Negishi, H.

Nerem, R. S.

Neumann, G. A.

Newitt, L.

Nishida, Y.

Nishigami, K.

Nishijima, T.

Nishimura, T.

Nishizawa, O.

Niu, F.

Noguchi, T.

Nosov, $\mathrm{M}$.

Nowack, R.

Obana, K.

Obara, K.

Obara, T.

O'Brien, T. P.

Ogata, Y.

Ohsiman, N.

Okada, Tomomi

Okada, Tatsuaki

Okada, Y.

Okamoto, T.

Okubo, Y.

Olsen, N.

Ondoh, $\mathrm{T}$

Ono, $\mathrm{T}$.

Opgenoorth, $\mathrm{H}$.

Otsuka, Y.

Oyama, K.

Pan, E.

Parrot, M.
Peng, Z.

Peyrat, S.

Phillips, S.

Piatanesi, A.

Pilipenko, S.

Pilkington, $\mathrm{M}$.

Ping, J.

Pitarka, A.

Pratt, T. L.

Pullian, J.

Purucker, M.

Rao, N. P.

Rasson, J. L.

Ratchkovski, N.

Rau, R.-J.

Rawland, D. E.

Raymond, C.

Reda, J.

Richards, P. G.

Rietbrock, A.

Rigoti, A.

Robion, $\mathrm{P}$.

Rogers, G.

Rycroft, M. J.

Rymer, M. J.

Sabaka, T. J.

Sabirats, J. J. C

Sager, W.

Sagiya, T.

Saito, A.

Saito, S.

Saito, T.

Sakai, S.

Sakai, Y.

Sakurai, T.

Saltus, R.

Santis, A. D.

Santolik, O.

Sasai, Y.

Sasaki, F.

Sastri, J. H.

Satake, K.

Sato, H.

Sato, K.

Sato, T.

Schott, J. J.

Seama, N.

Sekido, M.

Shen, Z.

Shibata, T.

Shibutani, T.

Shibuya, K.

Shimada, S.

Shimizu, H.

Shinohara, M.

Shiokawa, K.

Sibeck, D. G.

Simons, M.

Smith, A. K.

Smith, R.
Sneeuw, N.

Springer, A.

Stanley, M.

Stening, R. J.

Storini, M.

Strangeways, H.

Stutzmann, E.

Suetsusgu, D.

Summers, D.

Swenson, G. R.

Syndergaard, S.

Tabei, T.

Tadokoro, K.

Taguchi, S.

Takahashi, H.

Takahashi, T.

Takenaka, H.

Tanaka, A.

Tanimoto, $\mathrm{T}$.

Tanioka, Y.

Tarits, $\mathrm{P}$.

Taymaz, T.

Teisseyre, R.

Teunissen, $\mathrm{P}$.

Thio, H. K.

Thurber, C. H.

Titov, V. V.

Tivey, M. A.

Tobita, M.

Toda, S.

Tokunaga, $\mathrm{T}$.

Townend, J.

Tregoning, $\mathrm{P}$.

Triyoso, W.

Tsujii, T.

Tsumura, N.

Tsunomura, $\mathrm{S}$.

Tsutsumi, H.

Tyler, R.

Ueda, Y.

Uhira, K.

Utada, $\mathrm{H}$.

Uyeshima, M.

vander Lee, $\mathrm{S}$.

Vassiliadis, D.

Velasco, A. A.

Viljanen, A.

Vinnik, L.

Visser, P.

Wald, D. J.

Waldhauser, F.

Walker, R. T.

Wallace, L.

Wang, C.-Y.

Wang, $\mathrm{K}$.

Wannamaker, P.

Wardinski, I.

Waters, C. L.

Webb, T.

Weckmann, U. 
Weinstein, S. A.

Whaler, K.

White, A.

Williams, E. R.

Wing, S.

Wooster, M. J.

$\mathrm{Wu}, \mathrm{D}$.

$\mathrm{Wu}, \mathrm{Q}$.
$\mathrm{Xu}, \mathrm{P}$.

Yagi, Y.

Yamada, N.

Yamamoto, K.

Yamanaka, $\mathrm{H}$.

Yamauchi, T.

Yamazaki, T.

Yeoman, T. K.
Yokoyama, T.

Yokoyama, Y.

Yomogida, K.

Yoshida, S.

Yoshida, Y.

Yoshimoto, K.

Yoshioka, S.

Yurimoto, N.
Zahradnik, J.

Zhang, S.

Zhao, D.

Zhao, X.

Zhu, L. 